\title{
PROOF OF A CONJECTURE OF BANK AND LAINE REGARDING THE PRODUCT OF TWO LINEARLY INDEPENDENT SOLUTIONS OF $y^{\prime \prime}+A y=0$
}

\section{LI-CHIEN SHEN}

Dedicated to Professor A. Edrei on his 70 th birthday

\begin{abstract}
Let $A$ be a transcendental entire function of order $<1$. If $w_{1}$ and $w_{2}$ are two linearly independent solutions of the differential equation $y^{\prime \prime}+A y=0$, then at least one of $w_{1}, w_{2}$ has the property that the exponent of convergence of its zeros is $>1$.
\end{abstract}

1. Introduction. In this note we consider the differential equation

$$
y^{\prime \prime}+A(z) y=0,
$$

where $A$ is entire. Let $w_{1}$ and $w_{2}$ be two linearly independent solutions of the D.E. (1). Assume that $w_{1}$ and $w_{2}$ are normalized so that their Wronskian is identically 1. That this is possible is an obvious consequence of the homogeneous character of (1). Set $f=w_{1} w_{2}$. Bank and Laine [2] observed that the function $f$ satisfies the differential equation

$$
-4 A f^{2}=2 f f^{\prime \prime}-\left(f^{\prime}\right)^{2}+1
$$

From the above relation, it follows that $f$ has the property: If $z_{0}$ is a zero of $f$, then either $f^{\prime}\left(z_{0}\right)=1$ or $f^{\prime}\left(z_{0}\right)=-1$. Then we shall say that $f$ has the $B-L$ property at $z_{0}$.

If $f(z)$ has the $B$-L property at each one of its zeros, we simply say that $f(z)$ has the $B$ - $L$ property.

Using the Wiman-Valiron theory, Bank and Laine [2, p. 358] proved that if the order of $f$ is $<\frac{1}{2}$, then $f$ cannot have the B-L property.

Edrei [4] proved that if the growth of the Nevanlinna characteristic of an entire, transcendental function $f$ is sufficiently regular, then it is impossible for $f$ to be of order $<1$ and also possess the B-L property.

The main purpose of this note is to establish

Theorem 1 (Bank-Laine Conjecture). An entire function $f$, of order $<1$, cannot possess the $B-L$ property unless it is a polynomial of the form $a z^{2}+b z+c$ with $4 a c-b^{2}+1=0$.

Received by the editors November 26, 1984 and, in revised form, April 2, 1985.

1980 Mathematics Subject Classification. Primary 34A20, 30D35; Secondary 34D10, 34A30.

Key words and phrases. Linearly independent solutions, entire functions, Cartan's lemma, Carleman's differential inequality. 
Instead of proving Theorem 1 directly, we shall derive it from the following slightly more informative

THEOREM 2. Let $f$ be an entire function of order $\rho$, and of lower order $\lambda$, possessing the $B$-L property.

(i) If $f$ is transcendental, then $\rho+\lambda \geqslant 2$.

(ii) If $f$ is not transcendental, then $f$ is a polynomial of degree $\leqslant 2$, of the form $a z^{2}+b z+c$ with $4 a c-b^{2}+1=0$.

It should be noted that assertion (i) of the theorem is automatically satisfied if $\rho \geqslant 2$; it is therefore sufficient to carry out our proofs under the restriction $\rho<2$.

The following consequence of Theorem 2 is immediate.

COROLlaRY 1. Entire functions of order 1 possessing the B-L property must have regular growth (i.e., $\rho=\lambda$ ).

Theorem 1 may also be translated into an equivalent statement about differential equations.

Corollary 2. Let $A(z)$ be entire, transcendental, and of order $<1$, and let $w_{1}(z)$, $w_{2}(z)$ be two linearly independent solutions of $y^{\prime \prime}+A y=0$. Then at least one of them has zeros whose exponent of convergence is $>1$.

Our method is based on a consequence of Carleman's differential inequality, stated below as Arima's Theorem A.

Let $D=\{z:|f(z)|>1\}$. For any $r>0$, let $D_{r}$ be the part of $D$ lying in $|z|<r$. Let $A_{k}(r)(k=1,2, \ldots, n(r))$ be the arcs of $|z|=r$ contained in $D$, and let $r \theta_{k}(r)$ be their lengths. We define $\theta(r)=\infty$ if the entire circle $|z|=r$ lies in $D$. Otherwise, $\theta(r)=\max _{k} \theta_{k}(r)$.

TheOrem A (ARIMA). Let $f$ be entire, and let $D$ be the domain where $|f(z)|>1$. Let $\theta(r)$ be defined as above for the domain $D$. Then for any $0<\alpha<1$ we have

$$
\ln \ln M(r, f)>\pi \int_{r_{0}}^{\alpha r} \frac{d t}{t \theta(t)}-c\left(\alpha, r_{0}\right)
$$

where $0<r_{0}<\alpha r$ and $c\left(\alpha, r_{0}\right)$ is independent of $r$.

The proof can be found in Arima's paper [1, p. 64].

REMARK. Without altering Arima's proof, it is easily seen that if $f$ is an analytic function, single-valued and regular in a region $|z|>R_{0}>0$, and if, as $r \rightarrow \infty$,

$$
\max _{|z|=r}|f(z)|=M(r, f) \rightarrow \infty, \quad r>R_{0},
$$

then the inequality (3) of Theorem A continues to hold provided $r_{0}$ is large enough.

Our proof requires this slightly extended version of Arima's theorem.

2. Functions with the B-L property. The following two lemmas characterize functions with the B-L property.

LEMMa 2.1. Let $f$ be an entire function. Define

$$
h(z)=1 / f^{2}-\left(f^{\prime} / f\right)^{2}+2 f^{\prime \prime} / f .
$$

If $f$ has the $B-L$ property, then $h$ is entire. 
LEMMA 2.2. Let $f$ be an entire function with the B-L property. Then $f=w_{1} w_{2}$, where $w_{1}$ and $w_{2}$ are linearly independent solutions of the D.E. (1) with $A$ defined as in (2).

The proofs of both lemmas can be found in Bank and Laine's paper [3, p. 666].

3. Estimates for $f^{\prime \prime} / f$ and $f^{\prime} / f$. The following lemma plays an essential role in the proof of Theorem 2 .

LEMMA 3.1. Let $f$ be entire, of order $\rho, \rho<2$, and $I_{k}=\left(2^{k}, 2^{k+1}\right)$. Then for all $k$ large, say $k>k_{0}$, there exists a set $E_{k}$ in $I_{k}$ which is a finite union of subintervals, and such that

$$
\mu\left(E_{k}\right)<(4 / 5 k \ln 2) \mu\left(I_{k}\right)
$$

and

$$
\left|\frac{f^{\prime \prime}\left(r e^{i \theta}\right)}{f\left(r e^{i \theta}\right)}\right|+\left|\frac{f^{\prime}\left(r e^{i \theta}\right)}{f\left(r e^{i \theta}\right)}\right|^{2}<M r^{6}, \quad 0 \leqslant \theta<2 \pi,
$$

if $r \in I_{k}-E_{k}$, where $M$ is a constant independent of $k$.

(By $\mu(A)$ we denote the linear length of the set $A$.)

Proof. Write

$$
f(z)=K z^{N} e^{c z} \Pi\left(1-\frac{z}{a_{n}}\right) e^{z / a_{n}}, \quad 0<\left|a_{1}\right| \leqslant\left|a_{2}\right| \leqslant\left|a_{3}\right| \leqslant \cdots .
$$

Differentiating $f$ logarithmically and noting the identity $f^{\prime \prime} / f=\left(f^{\prime} / f\right)^{\prime}+\left(f^{\prime} / f\right)^{2}$, we obtain

$$
\begin{gathered}
\frac{f^{\prime}}{f}=c+\frac{N}{z}+\sum_{j=1}^{\infty} \frac{1}{a_{j}}+\frac{1}{z-a_{j}}, \\
\frac{f^{\prime \prime}}{f}=\left(\frac{f^{\prime}}{f}\right)^{2}-\frac{N}{z^{2}}-\sum_{j=1}^{\infty} \frac{1}{\left(z-a_{j}\right)^{2}} .
\end{gathered}
$$

Let $n(t)$ be the usual counting function of the zeros of $f$. Since the order of $f$ is $<2$, we have, for all $t$ large, say $t>t_{0}, n(t)<t^{\xi}$, where $\rho<\xi<2$.

Choose an integer $k_{0}$ so that $2^{k_{0}} \geqslant t_{0}$. For an integer $k \geqslant k_{0}$, let $r_{k}=2^{k}$ and $m=n\left(3 r_{k}\right)$. Then

$$
m<\left(e r_{k}\right)^{\xi}
$$

We now confine $z$ to the annulus

$$
r_{k} \leqslant|z| \leqslant r_{k+1} .
$$

We note that (3.3) yields

$$
\frac{f^{\prime}}{f}=z \sum_{j=1}^{m} \frac{1}{a_{j}\left(z-a_{j}\right)}+R_{1}(z)
$$

with

$$
\left|R_{1}(z)\right| \leqslant 3|z| \sum_{j=m+1}^{\infty} \frac{1}{\left|a_{j}\right|^{2}}+|c|+\frac{N}{|z|}=o(|z|)
$$


Similarly, from (3.4)

$$
\frac{f^{\prime \prime}}{f}=\left(\frac{f^{\prime}}{f}\right)^{2}-\sum_{j=1}^{m} \frac{1}{\left(z-a_{j}\right)^{2}}+R_{2}(z)
$$

with

$$
\left|R_{2}(z)\right| \leqslant 9 \sum_{j=m+1}^{\infty} \frac{1}{\left|a_{j}\right|^{2}}+\frac{N}{|z|^{2}}=O(1) .
$$

To complete our estimate we use Cartan's lemma in geometrical form. Set

$$
d=r_{k} / 5 \log r_{k}, \quad k \geqslant k_{0} .
$$

Then by Cartan's lemma there exist $p$ disks, $p \leqslant m$. (See [5, pp. 20-21].)

$$
D_{j}=\left\{z:\left|z-a_{j}\right| \leqslant d_{j}\right\}, \quad j=1,2, \ldots, p,
$$

such that $\sum_{j=1}^{p} d_{j}=2 d$ and such that, if $z \notin \bigcup_{j=1}^{p} D_{j}$, it is possible to find a renumbering $a_{1}^{\prime}, a_{2}^{\prime}, \ldots, a_{m}^{\prime}$ of $a_{1}, a_{2}, \ldots, a_{m}$, satisfying

$$
\left|z-a_{j}^{\prime}\right| \geqslant j d / m, \quad j=1,2, \ldots, m \text {. }
$$

(The renumbering may depend on $z$.)

From (3.13), (3.5), and (3.6), we obtain

$$
\begin{gathered}
\left|\sum_{j=1}^{m} \frac{z}{a_{j}\left(z-a_{j}\right)}\right| \leqslant|z|\left(\frac{m}{d}\right) \sum_{j=1}^{m} \frac{1}{\left|a_{j}\right|}=\frac{m|z|}{d} \int_{0}^{3 r_{k}} \frac{d n(t)}{t}=o\left(|z|^{3}\right) . \\
\left|\sum_{j=1}^{m} \frac{1}{\left(z-a_{j}\right)^{2}}\right|=O\left(\frac{m^{2}}{d^{2}}\right)=o\left(|z|^{2}\right)
\end{gathered}
$$

for $r_{k}<|z|<r_{k+1}$ and $z \notin \bigcup_{j=1}^{p} D_{j}$.

Let $A_{j}$ be the annulus generated by revolving the disks $D_{j}$ around the origin, and let $E_{k}^{*}$ be the intersection of $\bigcup_{j=1}^{p} A_{j}$ with the positive real axis. Now let

$$
E_{k}=\left(2^{k}, 2^{k+1}\right) \cap E_{k}^{*} \text {. }
$$

Since the sum of the diameters of $D_{j}$ is $4 d$, the length of $E_{k}$ is not greater than $4 d$. This proves (3.1).

Clearly, (3.2) follows from (3.7)-(3.10), (3.14), and (3.15).

For each $k \geqslant k_{0}$ we can construct a set $E_{k}$ in the above manner. We then define $E=\cup_{k=k_{0}}^{\infty} E_{k}$ and $\tilde{E}=\left\{r e^{i \theta}: r \in E ; 0 \leqslant \theta \leqslant 2 \pi\right\}$.

From (3.1) we easily derive

LEMMA 3.2. Let $r_{0}$ be any fixed positive number and, for each $r>0$, let $J_{r}$ be the set defined as $J_{r}=\left[r_{0}, \alpha r\right]-E, 0<\alpha<1$. Then

$$
\lim _{r \rightarrow \infty} \frac{1}{\ln r} \int_{J_{r}} \frac{1}{t} d t=1 .
$$

4. Proof of Theorem 2. Since $f$ has the B-L property, Lemma 2.1 implies that the function $h$ defined as (2.1) is entire. And since the order of $f$ is $\rho$, the order of $h$ must also be $\leqslant \rho$. Now there are two cases to be considered. We first consider 
Case 1. $h$ is a polynomial of degree $N$. From Lemma 2.2, $f$ is a product of two linearly independent solutions of D.E.

$$
y^{\prime \prime}-\frac{1}{4} h y=0 .
$$

Using Wiman-Valiron's theory of the maximum term, Bank and Laine [2, p. 354] observed that if the degree $N \geqslant 1$, then

$$
\ln M(r, f)=c_{1} r^{(N+1) / 2}(1+o(1)) \quad \text { as } r \rightarrow \infty,
$$

for some constant $c_{1}>0$. From (4.2) we conclude that $f$ has regular growth, that is, $\rho=\lambda=(N+2) / 2$. Thus $\rho+\lambda=N+2 \geqslant 3$. We now consider the case $N=0$. Then $h$ is constant. It is easy to see that if $h$ is a nonzero constant, then either $f$ is a nonzero constant or $f$ satisfies (4.2) with $N=0$, and this implies that $\rho+\lambda=2$. If $h=0$, then $(2.1)$ becomes

$$
2 f f^{\prime \prime}+1-\left(f^{\prime}\right)^{2}=0
$$

To solve $f$, we differentiate (4.3) and find $f f^{\prime \prime \prime}=0$. This implies that either $f$ is identically zero or $f$ is a polynomial of degree $\leqslant 2$. Since $f$ satisfies (4.3), $f$ has to be of the form $a z^{2}+b z+c$ with $4 a c-b^{2}+1=0$.

We now turn to

Case 2. $h$ is transcendental. Let

$$
D_{1}=\{z:|f(z)|>1\}, \quad D_{2}=\left\{z:\left|h(z) / z^{7}\right|>1\right\},
$$

and let $\theta_{i}(r)$ be defined as in $\S 1$ for the domain $D_{i}, i=1,2$. From (2.1) and (3.2) we deduce that for $z \in D_{1}-\tilde{E}$,

$$
|h(z)|<1+M r^{6}<r^{7}, \quad|z|=r>r_{0} .
$$

On the other hand, for $z \in D_{2}-\tilde{E}$ we have

$$
|h(z)|>h^{7} .
$$

Therefore, from (4.4) and (4.5) we deduce

LEMMA 4.1. Let $\theta(r)=\theta_{1}(r)+\theta_{2}(r)$. Then

$$
\theta(r)<2 \pi
$$

and

$$
\theta_{1}(r) \theta_{2}(r) \leqslant \theta^{2}(r) / 4
$$

for $r \geqslant r_{0}$ and $r \notin E$.

In view of the following remark, we may assume that $\theta_{1}(r) \theta_{2}(r) \neq 0$ for all $r \geqslant r_{0}$ and $r \notin E$.

REMARK. If there exists a sequence $\left\{t_{n}\right\}$ tending to infinity such that

(i) $\theta_{1}\left(t_{n}\right)=0$, then $f$ is clearly a constant, and from (2.1) $h$ is a constant;

(ii) $\theta_{2}\left(t_{n}\right)=0$, then $h$ is a polynomial.

We have already discussed this in Case 1. 
From Theorem A and the remark following it we have

$$
\begin{gathered}
\ln \ln M(r, f)>\pi \int_{r_{0}}^{\alpha r} \frac{d t}{t \theta_{1}(t)}-k_{1}, \\
\ln \ln M\left(r, \frac{h}{z^{7}}\right)>\pi \int_{r_{0}}^{\alpha r} \frac{d t}{t \theta_{2}(t)}-k_{2} .
\end{gathered}
$$

Therefore, it follows from (4.6)-(4.9) that

$$
\begin{array}{r}
\ln \ln M(r, f)+\ln \ln M\left(r, \frac{h}{z^{7}}\right)>\pi \int_{r_{0}}^{\alpha r} \frac{\theta(t)}{\theta_{1}(r) \theta_{2}(r)} \frac{d t}{t}+k \\
>4 \pi \int_{J_{r}} \frac{1}{t \theta(r)} d t+k>2 \int_{J_{r}} \frac{d t}{t}+k, \quad k=-k_{1}-k_{2} .
\end{array}
$$

From Lemma 3.2 and (4.10), we conclude immediately that

$$
\begin{aligned}
\rho+\lambda & \geqslant \lim _{r \rightarrow \infty} \frac{\ln \ln M(r, f)}{\ln r}+\varlimsup_{r \rightarrow \infty} \frac{\ln \ln M(r, h)}{\ln r} \\
& \geqslant \underset{r \rightarrow \infty}{\lim }\left(\ln \ln M(r, f)+\ln \ln M\left(r, h / z^{7}\right)\right) / \ln r \\
& \geqslant 2 .
\end{aligned}
$$

Combining the conclusions of both cases, we establish Theorem 2.

We now prove Corollary 2. Let $\rho_{A}$ be the order of the function $A(z)$. By assumption $\rho_{A}<1$. Let the Wronskian of $w_{1}$ and $w_{2}$ be normalized so that it is identically one. Then the product $f=w_{1} w_{2}$ satisfies the differential equation (2). This implies that the order of $f$ is $\geqslant \rho_{A}$. Let $\rho$ be the order of $f$ and we assume that $\rho \leqslant 1$. Replacing the function $h$ in Case 2 of Theorem 2 by $-4 A$ and repeating the same argument we conclude that $\rho_{A}+\rho \geqslant 2$. But this is incompatible with the assumptions that $\rho \leqslant 1$ and $\rho_{A}<1$. Therefore the order of $f$ must be $>1$. This corollary follows immediately from the estimate [2, p. 354, (8)]

$$
T(r, f)=O(N(r, 1 / f)+T(r, A)+\ln r) \quad \text { n.e. as } r \rightarrow \infty .
$$

REMARK. It has to be pointed out that to derive (4.2) it is absolutely essential that $h$ be a polynomial. If $h$ is replaced by a rational function, then (4.2) is no longer true. (For a counterexample see [2, p. 355].)

5. An example. There are functions of order $<1$ that have the B-L property at all but one of their zeros.

One such example is the function $f(z)=2 \sqrt{z} \sin \sqrt{z}$. It is entire, of order $1 / 2$, and with the exception of $z=0$, where $f(0)=0$ and $f^{\prime}(0)=2$, the B-L property is satisfied at all the other zeros of $f$. By substituting this function into (2.1) we see that $f$ satisfies the D.E.

$$
2 y^{\prime \prime} / y-\left(y^{\prime} / y\right)^{2}+1 / y^{2}=-(z+3) / 4 z^{2}=h(z) .
$$

Note that in this case $h$ is a rational function with a pole at $z=0$. From the proof of Lemma 2.1 it is easy to see that the existence of this pole at $z=0$ is caused by the failure of the B-L property at this point. 
Using the method in the last section, we can derive the following

Proposition. Let $f$ be an entire function of order $<1$ and let $f(0)=0$. Assume that $f$ has the B-L property at all its zeros with the exception of $z=0$, where $f^{\prime}(0)=2$. If the power series of $f$ is $f(z)=2 z+c_{2} z^{2}+\cdots, c_{2} \neq 0$, then

$$
f(z)=\frac{2}{\sqrt{-3 c_{2}}} z^{1 / 2} \sin \sqrt{-3 c_{2} z} .
$$

We note that if $c_{2}=0$, then $f(z)=2 z$.

OUtline OF THE Proof. A straightforward substitution of $f$ into (2.1) yields

$$
2 f^{\prime \prime} / f-\left(f^{\prime} / f\right)^{2}+1 / f^{2}=-\frac{3}{4}\left(z^{-2}-c_{2} z^{-1}\right)+h_{1},
$$

where $h_{1}$ is an entire function.

Since the order $\rho$ of $f$ is $<1$, we can improve the estimate of (3.2) by choosing $\xi$ in $(3.5)$ to be $(1+2 \rho) / 3$. We then obtain

$$
\left|\frac{f^{\prime \prime}\left(r e^{i \theta}\right)}{f\left(r e^{i \theta}\right)}\right|+\left|\frac{f^{\prime}\left(r e^{i \theta}\right)}{f\left(r e^{i \theta}\right)}\right|<M r^{\rho-1}, \quad r \notin E .
$$

Repeating the argument used in Case 2 , we conclude that $h_{1}$ cannot be transcendental. From (5.1) and (5.2) we deduce further that $h_{1}=0$. Therefore, $f$ satisfies the D.E.

$$
2 y^{\prime \prime} / y-\left(y^{\prime} / y\right)^{2}+1 / y^{2}=-\frac{3}{4}\left(z^{-2}-c_{2} z^{-1}\right) \text {. }
$$

One way to obtain a general solution of the above equation is to consider the D.E.

$$
w^{\prime \prime}+\frac{3}{16}\left(z^{-2}-c_{2} z^{-1}\right) w=0 .
$$

Since the coefficient of $w$ in (5.4) is a rational function with a pole at $z=0$, the solutions of this D.E. are no longer entire. In fact, by direct substitution it is easily verified that

$$
\begin{aligned}
& w_{1}^{*}=z^{1 / 4} \cos \left(\sqrt{-3 c_{2} z} / 2\right), \\
& w_{2}^{*}=z^{1 / 4} \sin \left(\sqrt{-3 c_{2} z} / 2\right),
\end{aligned}
$$

are two linearly independent solutions of the D.E. (5.4).

If $w_{1}$ and $w_{2}$ are any two linearly independent solutions of this D.E., and if they are normalized so that their Wronskian is 1 , then $f=w_{1} w_{2}$ satisfies (5.3). Conversely, if $f$ satisfied (5.3), then $f$ is a product of two normalized linearly independent solutions of (5.4).

Hence $f=\left(4 / \sqrt{-3 c_{2}}\right) w_{1}^{*} w_{2}^{*}$ is a solution of the D.E. (5.3). From (5.5) and (5.6) we see that this function is the only solution which is entire. (All of the other solutions have a branch point at $z=0$.) It is easy to verify that $f$ has all the required properties, and the proof is complete.

ACKNOWLedgement. I would like to express my deep gratitude to Professor A. Edrei. Many of the ideas contained in this paper are motivated by his unpublished manuscript. 


\section{BIBLIOGRAPHY}

1. K. Arima, On maximum modulus of integral functions, J. Math. Soc. Japan 4 (1952), 62-66.

2. S. Bank and I. Laine, On the oscillation theory of $f^{\prime \prime}+A f=0$ where $A$ is entire, Trans. Amer. Math. Soc. 273 (1982), 351-363.

3. On the zeros of meromorphic solutions of second order linear differential equations, Comment. Math. Helv. 58 (1983).

4. A. Edrei, The problem of Bank and Laine, unpublished manuscript.

5. B. Ja. Levin, Distribution of zeros of entire functions, Transl. Math. Monos., vol. 5, Amer. Math. Soc., Providence, R. I., 1963.

Department of Mathematics, Syracuse University, Syracuse, New York 13210

Current address: Department of Mathematics, 201 Walker Hall, University of Florida, Gainesville, Florida 32611 